\title{
Design of Intelligent Home Application system based on CC2530
}

\section{Yuanyuan $\mathrm{Hu}^{1, \mathrm{a}^{*}}$, Xue $\mathrm{Wu}^{1, \mathrm{~b}}$ and Xining $\mathrm{Li}^{1, \mathrm{c}}$}

\author{
${ }^{1}$ Li Shi Economic and technological Development Zone Fushun City, Liaoning, P.R.China \\ a281011796@qq.com, b916192994@qq.com, ‘291648643@qq.com
}

Keywords: CC2530; Z-Stack; sensor; ZigBee; Unicast; Intelligent Home

\begin{abstract}
Intelligent Home is the embodiment of linking under the influence of Internet. The intelligent home connects all kinds of equipment in the home together to provide home appliance control, illuminating control, remote control of telephone, indoor and outdoor remote control, burg proof alarm, environmental supervision Measurement, HVAC control, infrared forwarding, programmable timing control and other functions and means. In this paper, a set of intelligent home system is designed based on ZigBee chip CC2530.The system has low power consumption and high precision, and can be popularized in modern home design.
\end{abstract}

\section{System Overall Design.}

Requirement analysis. Through investigation and investigation, the functions of the system should include the following aspects: intelligent lighting control, intelligent management of the whole house lighting, remote control and other intelligent control methods can be used to realize the remote control switch and dimming of the whole house lighting. The realization of various one-button lighting scene effects, such as full opening and closing, "Parlor, Cinema" and so on, can be realized by various control methods, such as timing control, telephone remote control, computer local and Internet remote control and so on. In order to achieve intelligent lighting energy-saving, environmental protection, comfort, convenient function. [1]

Design goal. From both residential and home levels, providing a vision of safety, energy conservation, health (sunshine and air), good communication (various means of communication), comfort and convenience (automation), With appropriate products and software, Provide linkage or integration of various systems. The system should be thorough and minimized the impact on the daily life of residents, to ensure that the privacy of residents in indoor activities is not infringed. [2]

Home control system, security requirements, lighting, Audio, telephone and other systems, with the life of the scene of the one-click instruction (such as entering, dining, welcome, sleep, short or long-term leave, etc.) as the core, the implementation of "one-click" control-including the linkage between the system control; The home security system focuses on solving the relationship between alarm sensitivity and minimization of false positives, for example, distinguishing the host from the intruder by programming the sensor or automatically identifying the identity; Home security system should include fire alarm system. [3]Emphasis on the ease of use of products and systems, simplification: the purpose of application of science and technology should make operation, programming and proportion imbalance easier, more economical, not more cumbersome; In particular, the operation of domestic equipment. Attach importance to the reliability of products and systems: select products that have been proven to be practical and reliable, and do not use software or equipment temporarily developed to meet the requirements of this project; Expansibility: limited to the schedule of planning and design and expected area expansion of the project, each system should have more than $100 \%$ expansion ability. 


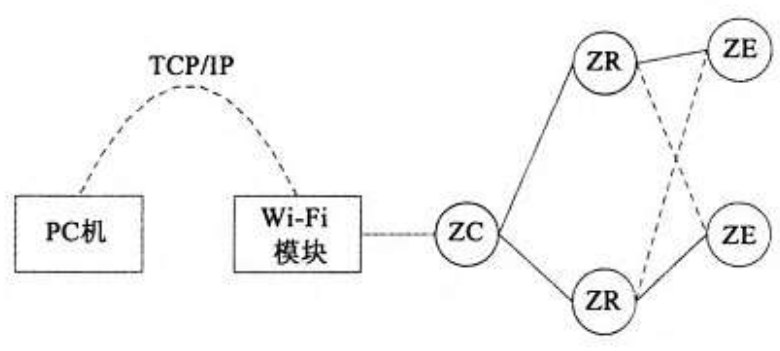

Figure 1. Hardware topology

\section{Hardware structure design.}

Software structure design. The software uses the Zstack protocol stack designed by TI for CC series chips produced by TI. The protocol stack conforms to the ZigBee specification, uses the idea of operating system to construct, adopts the time rotation mechanism, and provides multi-task processing mechanism. It can automatically realize the functions of building network, human network, distributed address assignment, message sending / receiving and so on. The subroutine is the substantive function of each module. The main subroutines include temperature acquisition, keyboard scanning and LCD display.

\section{Hard ware design.}

Hardware option. The hardware of this intelligent home control system is mainly composed of S3C44B0X main controller unit CC2530 wireless transmission module and MC39I GPRS. Hardware selection is based on CC2530 minimum system design for sensor nodes. Mainly contains temperature and humidity, human body proximity, lighting control, and door and window motor control CC2530 sampling and control node circuit. The wireless transmission of each node signal is realized by CC2530 module. CC2530 Coordinator sent to CC2530 to realize data Exchange between UART Port and S3C44B0X Master Controller. Information display function S3C44B0X is realized through the connection of SIO synchronous Serial Port and liquid Crystal screen of Diwen liquid Crystal Industry You can connect to the MC39I GPRS module via the UART interface, The AT command supported in MC39I is used to realize the remote control and remote view of the system.

\section{Hardware function module.}

Operational principle. As shown in figure 2, when infrared is transmitted and received from the tube, the circuit is switched on, and the comparator + terminal is equivalent to direct grounding, that is, the output of the $0 \mathrm{~V}$ voltage is $2.5 \mathrm{~V}$ voltage adjusted, so the OUT end of the comparator outputs low level. When there is an object blocking the infrared ray, the receiver barrier of the infrared tube is disconnected, and the comparator + terminal is connected to the VCCs, that is, $5 \mathrm{~V}$ voltage, so the comparator OUT terminal outputs high level and generates the detection signal. Access to the ZigBee infrared detection module P1.4 port. 


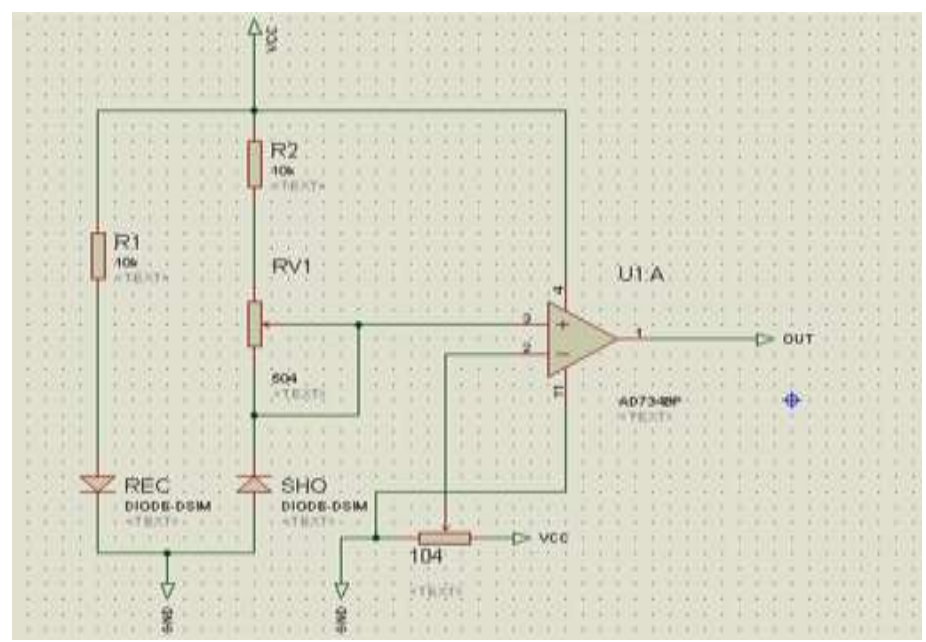

Figure 2. Principle diagram of infrared detection circuit

Smoke sensor module. Smoke sensor is to monitor the concentration of smoke to achieve fire prevention, smoke alarm inside the use of ionic smoke sensor, ion smoke sensor is a kind of advanced technology, stable and reliable work of the sensor, Widely used in various fire alarm systems, the performance is far better than the gas resistance type of fire alarm.
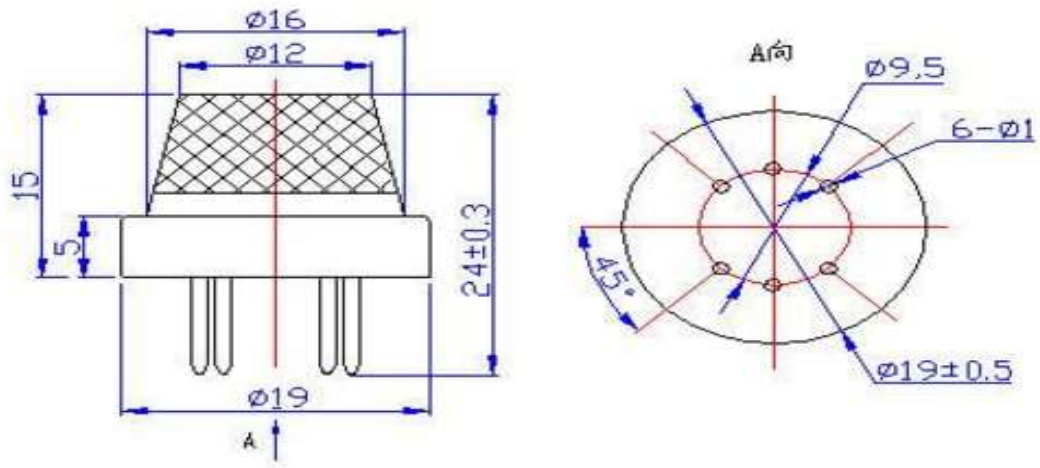

Figure 3. Principle of photosensitive resistance sensor module

In this system, we let the sensor node send its own data or status to the coordinator every $60 \mathrm{~s}$. After receiving the data, the coordinator sends it to the host computer serial port assistant through uart protocol. Serial port assistant display, the character St after the two nodes for the network address. The fifth bit is the room number, the sixth bit is the sensor type, the next four bits are the sensor's data or status, and the last one is the Terminator. When you want to control the lights or curtains of a room separately, by looking at the room and sensor type, copying its network address to fill in the command, through the serial port to the coordinator, coordinator by viewing its network address, unicast to the node.

Software design. The CC2530 development environment software developed by TI Company is IAR Embedded Workbench for 8051. The development environment has good man-machine interface and intelligent programming method, which is convenient to CC2530 online simulation. It's a great help for CC2530 developers.

The business process of the smart home system includes the business interaction between the PC software smart client and the Main, and the Main itself also has the function of monitoring the temperature and monitoring the infrared. After the system is powered on, Execute Main software, start smart client software on PC.

\section{Summary}

Intelligent home system is to use advanced computer technology, network communication technology, integrated wiring technology, medical and electronic technology in accordance with ergonomics principles, the integration of individual needs, will be related to the home life of various 
subsystems such as security, Lighting control, curtain control, gas valve control, information appliances, scene linkage, floor heating, health care, sanitation and epidemic prevention, security, and so on are organically integrated together, through the network comprehensive intelligent control and management, Realize "people first" the brand-new home life experience.

\section{References}

[1] Francis daCosta: Reconstructing the Future of the Internet of things (Renmin University of China Press, China 2016),p.15(In Chinese)

[2] R.Yang: Technology and Application of CC2530 single Chip computer (Machinery Industry Press , China 2016),p.13(In Chinese)

[3] X.An: The Development of Python in the Internet of things (Electronic industry publishing house, China 2018),p.57(In Chinese) 